\title{
Biofunctionalization of porcine-derived collagen matrices with platelet rich fibrin: influence on angiogenesis in vitro and in vivo
}

\author{
Sebastian Blatt ${ }^{1,2}$ (D) Valentin Burkhardt ${ }^{1} \cdot$ Peer W. Kämmerer $^{1} \cdot$ Andreas M. Pabst $^{3} \cdot$ Keyvan Sagheb $^{1}$. \\ Martin Heller ${ }^{4} \cdot$ Bilal Al-Nawas $^{1} \cdot$ Eik Schiegnitz $^{1}$
}

Received: 25 September 2019 / Accepted: 15 January 2020 / Published online: 6 February 2020

(C) The Author(s) 2020

\begin{abstract}
Objectives Porcine-derived collagen matrices (CM) can be used for oral tissue regeneration, but sufficient revascularization is crucial. The aim of this study was to analyze the influence of platelet-rich fibrin (PRF) on angiogenesis of different CM in vitro and in vivo.

Materials and methods Three different $\mathrm{CM}$ (mucoderm ${ }^{\circledR}$, jason $\circledast$, collprotect $\left.{ }^{\circledR}\right)$ were combined with PRF in a plotting process. Growth factor release (VEGF, TGF- $\beta$ ) was measured in vitro via ELISA quantification after 1,4 and 7 days in comparison to PRF alone. In ovo yolk sac (YSM) and chorion allantois membrane (CAM) model, angiogenic potential were analyzed in vivo with light- and intravital fluorescence microscopy after $24 \mathrm{~h}$, then verified with immunohistochemical staining for CD105 and $\alpha$ SMA. Results Highest growth factor release was seen after $24 \mathrm{~h}$ for all three activated membranes in comparison to the native CM (VEGF 24 h: each $p<0.05$; TGF- $\beta$ : each $p<0.001$ ) and the PRF (no significant difference). All activated membranes revealed a significantly increased angiogenic potential in vivo after $24 \mathrm{~h}$ (vessels per $\mathrm{mm}^{2}$ : each $p<0.05$; branching points per $\mathrm{mm}^{2}$ : each $p<$ 0.01 ; vessel density: each $p<0.05$ ) and with immunohistochemical staining for CD105 (each $p<0.01)$ and $\alpha \operatorname{SMA}(\operatorname{each} p<0.05)$. Conclusions PRF improved the angiogenesis of $\mathrm{CM}$ in vitro and in vivo.

Clinical relevance Bio-functionalization of CM with PRF could easily implemented in the clinical pathway and may lead to advanced soft tissue healing.
\end{abstract}

Keywords Angiogenesis · Vascularization · Collagen matrices $\cdot$ Platelet-rich fibrin $\cdot$ Tissue engineering

\section{Introduction}

Porcine-derived collagen matrices (CM) are widely used in oral surgery, periodontology and implantology for hard and

Electronic supplementary material The online version of this article (https://doi.org/10.1007/s00784-020-03213-8) contains supplementary material, which is available to authorized users.

Sebastian Blatt

sebastian.blatt@unimedizin-mainz.de

1 Department of Oral- and Maxillofacial Surgery, University Medical Center, Augustusplatz 2, 55131 Mainz, Germany

2 Platform for Biomaterial Research, Biomatics Group, University Medical Center, Langenbeckstrasse 1, 55131 Mainz, Germany

3 Department of Oral- and Maxillofacial Surgery, Federal Armed Forces Hospital, Rübenacher Str. 170, 56072 Koblenz, Germany

4 Department of Gynecology, University Medical Center, Langenbeckstrasse 1, 55131 Mainz, Germany soft tissue regeneration [1]. Principally, there are two different indications in the clinical routine: on the one hand, CM can be used as soft tissue grafts as a possible alternative to autogenously connective tissue [2]. Derived from porcine pericardium, peritoneum or dorsal skin, some CM can create different compartments for defect healing. As an occlusive barrier, they prevent the ingrowth of gingival soft tissue into a periodontal or bone defect and therefore allow the unimpeded proliferation and differentiation of osteogenic progenitor cells that lead to tissue regeneration [3]. All CM are decellularized, deproteinized and avascular, but differ in regard to their resorption process [4]. Therefore, defect healing relies heavily on the biomechanics of the used membrane.

Furthermore, angiogenesis and the formation of newly formed blood vessels is mandatory to provide the required nutritions, oxygen, immunological cells, mesenchymal stem cells, and growth factors especially in the initial healing phase [5]. Problematically, the CM might primarily exclude the new blood vessels as well as the ingrowth of osteoprogenitor cells from the periosteum. In order to overcome this limitation, 
biofunctionalization of the dehydrated, acellular and avascular CM might lead to a optimized wound healing for example via growth factor loading [6].

Autologous platelet concentrates represent a possible way to extract the patient's growth factors of the peripheral blood that could be used for further biofunctionalization. Developed as an advanced version of the fibrin sealants more than 40 years ago, nowadays different concentrates were defined that differ in cell content and matrix architecture as well as centrifugation process, among them Platelet- Rich Fibrin [7]. In this context, Park et al. modified a CM inter alia by adding PRF and found a significantly enhanced endothelial cell migration in vitro [8].

Since the introduction of the PRF in 2001 by Choukroun et al., it is widely used to support oral soft tissue regeneration [9]. In the literature, there is evidence that PRF can be successfully used in the therapy of gingival recession or intrabony defects in periodontology $[10,11]$, in reducing pain and swelling after third molar removal [12], implant stability [13] and other aspects of regenerative medicine [14, 15]. It is chair side produced via specific centrifugation after venous blood collection and can be processed as a liquid, injectable or stable PRF respectively. PRF can be used as a biologically active membrane that facilitates wound healing [16]. Vascular Endothelial Growth Factor (VEGF) mainly triggers the proliferation and differentiation of endothelial cells. However, different cell-cell interactions trigger a complex interplay with other important factors such as Transforming Growth FactorBeta1 (TGF- $\beta 1$ ) and Platelet-derived Growth Factor (PDGF). Here, mainly paracrine actions lead to the development of a vascular system [17]. As a result, enhanced microvessel-like structure formation was shown when endothelial cells were cultured with PRF in comparison to negative control [18]. In comparison to other autologous thrombocyte concentrates such as Platelet Rich Plasma (PRP) the growth factor release is described gradually and mimics physiological conditions. As a result, the angiogenesis may initiate and tissue repair can be optimized [19].

The aim of the present study was to use PRF to biologically activate different $\mathrm{CM}$.

The hypothesis was that activated membranes show similar pro-angiogenic potential in comparison to pure PRF in vitro and in vivo. Since differentially structured CM were used it was secondly to be analyzed which collagen composition supports bio-activation via PRF the best.

\section{Material and methods}

\section{Sample preparation}

Three different commercially available porcine-derived collagen matrices $(\mathrm{CM})$ were used: mucoderm ${ }^{\circledR}$, jason ${ }^{\circledR}$ and collprotect ${ }^{\circledR}$, all: $15 \times 20 \mathrm{~mm}$, botiss biomaterials $\mathrm{GmbH}$, Zossen, Germany). Mucoderm ${ }^{\circledR}$ is a porcine, native, dermal three-dimensional collagen matrix without any artificial crosslinking that contains natural collagen type I and III. Similarly, collprotect ${ }^{\circledR}$ matrix represents a native, dermal threedimensional collagen matrix on naturally collagen and elastin [20]. The CM of porcine pericardium (jason ${ }^{\circledR}$ ) displays interconnected surface system of pores [4].

\section{Platelet-rich fibrin}

For the PRF protocol, blood was collected from three healthy volunteers that gave informed consent to this study. After punction of the cephalic vein or the medial median cubital vein, $10 \mathrm{ml}$ venous blood was collected with the vacutainer system and specific sterile plain vacuum tubes with additional silicone within their coating surface (A-PRF+, Mectron, Carasco, Italy). Next, the collected blood was directly processed with the slow centrifugation protocol (1200 rpm for $8 \mathrm{~min}$, relative centrifugal force $177 \mathrm{~g}$ at a fixed angle rotor with a radius of $110 \mathrm{~mm}$, Duo centrifuge, Mectron, Carasco, Italy) as previously described [21]. After centrifugation, the PRF was pressed with the "PRF Box" (Mectron, Carasco, Italy) as indicated by the manufacturer. Next, PRF was cutted in small pieces of 16-25 $\mathrm{mm}^{2}$ and replaced in the "PRF box". Subsequent, the respective CM was cutted in the same sizes and placed above the PRF, then plotted together for $30 \mathrm{~s}$ within the box manually.

Following membranes ( $n=8$ each) were analyzed: $\mathrm{JB}=$ activated jason ${ }^{\circledR}$ membrane, $\mathrm{JN}=$ native jason ${ }^{\circledR}$ membrane, $\mathrm{MB}=$ activated mucoderm ${ }^{\circledR}$ membrane, $\mathrm{MN}=$ native mucoderm ${ }^{\circledR}$ membrane, $\mathrm{CB}=$ activated collprotect ${ }^{\circledR}$ membrane, $\mathrm{CN}=$ native collprotect ${ }^{\circledR}$ membrane, $\mathrm{PRF}=$ Platelet rich fibrin and YSM = native Yolk sac membrane. The activated membranes were compared with each other, their native equivalent as well with PRF and a negative control in the in vivo testing. For every experiment, PRF from all three donors were obtained, used for the activation process and analyzed grouped.

\section{ELISA assay for in vitro quantification of growth factor expression}

The respective membranes $(n=9$ per respective membrane, in total: $n=63$ ). were cut into pieces of 0,5 square $\mathrm{cm}$ and transferred into 6-well plates with $2 \mathrm{ml}$ of an eagle medium (Lonza, Cologne, Germany) without growth factors under sterile conditions and incubated at $37{ }^{\circ} \mathrm{C}$ and $5 \% \mathrm{CO}_{2}$. After 1,4 and 7 days the medium was changed. For each time point, $1.4 \mathrm{ml}$ of the medium was collected for further analysis and stored at $-80{ }^{\circ} \mathrm{C}$.

Next, the samples were analyzed by a VEGF (R\&D Systems, Minneapolis, USA) and a TGF- $\beta$ (R\&D Systems, 
Minneapolis, USA) enzyme linked immuno-sorbent assay (ELISA) using the protocol according to the manufacturer. The plates were measured at $450 \mathrm{~nm}$ with an ELISA plate reader (Molecular Devices, San Jose, USA) using the 'SoftMax Pro 5.4' (Molecular Devices, San Jose, USA) software.

\section{In ovo yolk sac membrane (YSM) assay for in vivo quantification of angiogenesis}

Fertilized white Leghorn chicken eggs (LSL Rhein-Main, Dieburg, Germany) were incubated at $38{ }^{\circ} \mathrm{C}$ at constant humidity in a special incubator (Janeschitz, Hammelburg, Germany). On day four of embryological development, the egg was prepared by removing $8-10 \mathrm{ml}$ egg white and cutting a $3 \times 3 \mathrm{~cm}$ window into the upwards pointing part of the egg under sterile conditions. On day 5, a 16$25 \mathrm{~mm}^{2}$ membrane $(n=28$ per respective membrane, in total: $n=224$ ), produced as described above, was applied with a forceps and incubated for further use under the same conditions. After $24 \mathrm{~h}$ pictures were taken at 30fold and 50-fold magnification before and after removing the membrane by centering the middle of the membrane with a digital microscope (KEYENCE, Neu-Isenburg, Germany) and analyzed with the respective software (KEYENCE, Neu-Isenburg, Germany). Here, the pictures were pseudonymized and a grid of $500 \mu \mathrm{m}$ side length was laid over. Afterwards all vessels and branching points of the vessels were counted in the region of interest per $\mathrm{mm}^{2}$. Afterwards, the embryos were euthanized by cutting the main vessels.

\section{In ovo chicken chorionallantoic membrane (CAM) assay for in vivo quantification of angiogenesis}

For CAM assay, fertilized white Leghorn chicken eggs were incubated at the same conditions and prepared on development day 4 of the embryo as mentioned above. Next, the eggs were further incubated until development day 11 , before the respective membrane of $0.5 \mathrm{~cm}^{2}$ was applied with a forceps and incubated for another $24 \mathrm{~h}$ under same conditions $(n=9$ per respective membrane, in total: $n=63$ ). Then, $0.1 \mathrm{ml}$ of a $5 \%$ FITC-dextran solution in $0.9 \% \mathrm{NaCl}$ (Merck, Darmstadt, Germany) was applied intravenously. Pictures were taken of the CAM at the upper border of the membrane (magnification: 5-fold) with an intravital fluorescence microscope (Olympus, Hamburg, Germany) and analyzed by the cellSens Dimension software (Olympus, Hamburg, Germany). Here, the pictures were pseudonymized and a grid of $500 \mu \mathrm{m}$ side length was used to count vessels and respective branching points in the region of interest. Furthermore the pictures were processed with software
Fiji (Image $\mathrm{J}$ ) to enhance the contrast of vessels and the background. After inverting the pictures, the plug-in "Vessel Analyzer" was used to quantify the vessel density. Therefore, 3 squares per picture of $0.5 \mathrm{~mm}$ side length at the edge of the membrane were chosen and vessel number and branching points counted. This way, vessel density was defined. Afterwards the embryos were euthanized as described previously.

\section{Histological preparation}

The area of the Yolk underneath the respective membrane was cut out and further analyzed immunohistochemistry. Therefore, samples ( $n=9$ per respective membrane, in total $n=72$ ) were immediately fixed in formaldehyde for $24 \mathrm{~h}$. The tissues were embedded in paraffin and histological sections of $5 \mu \mathrm{m}$ were made. They were stained with hematoxylin-eosin (Merck, Darmstadt, Germany) and analyzed via light microscopy (KEYENCE, Neu-Isenburg, Germany). The selected samples were furthermore stained with $\alpha$-SMA (SigmaAldrich, St. Louis, USA) and CD105 (Biorbyt, Cambridge, England) antibodies following the producer's instructions. Briefly, for the $\alpha$-SMA staining specimen were dewaxed and afterwards the proteins were unmasked in a steam cooker for $30 \mathrm{~min}$. They were treated with a peroxidase (Dako, Jena, Germany) and a protein block (Dako, Jena, Germany) before adding the $\alpha$-SMA antibody (diluted 1:1000). After one hour, incubation marked polymer-HRP anti mouse (Dako, Jena, Germany) was added for another $30 \mathrm{~min}$. Before rehydration the specimen were treated with DAB (Dako, Jena, Germany). In order to perform the CD 105 staining, specimen were dewaxed and treated with $0.1 \%$ Triton-X-100 (SigmaAldrich, St. Louis, USA) for 5 min. Blocking was performed by using PBS/BSA (Sigma-Aldrich, St. Louis, USA) and PBS/Goat normal serum (Dako, Jena, Germany). Afterwards the specimen were incubated with the CD 105 antibody (diluted 1:750) for one hour, followed by incubation with the secondary antibody alpha rabbit 488 (diluted 1:100, Invitogen, Carlsbad, USA) for an additional hour. Finally cell nucleus staining was performed, using DAPI (ThermoFischer, Waltham, USA). The incubation of the last two steps was made in a dark environment due to the fluorescent characteristics of the staining. After taking pictures (magnification: $\times 2$ ) of the whole specimen, they were pseudonymized for further data evaluation and analyzed by using the BZ-II Analyzer (KEYENCE, Neu-Isenburg, Germany) software. The pictures were overlaid with a grid of $0,25 \mathrm{~mm}$ side length. In 3 standardized squares defined as the region of interest, vessels were counted. Furthermore the sections, which were stained with CD105 and $\alpha$-SMA, were analyzed with the Hybrid Cell Count (KEYENCE, Neu-Isenburg, Germany) software and the percentage of histological stained tissue was measured. 


\section{Statistical analysis}

For data analysis, SPSS (Version 23, IBM, Ehningen, Germany) was used. Due to their reference to different subsets, the main questions were not adjusted to multiple testing. Before testing, all variables were evaluated for normal distribution with the Shapiro-Wilk test. The subsets of the YSM assay were tested by the Poisson-Regression. The subsets of ELISA testing were analyzed by an ANOVA-Regression. In case of normal distribution, student's T test was applied for further analysis. For non-normal distributed variables, the Mann-Whitney test was used and differences between all four groups detected via a student's T test. A $p$ value $\leq 0.05$ was defined as statistical significant. For data illustration, box plots were chosen.

\section{Results}

\section{ELISA testing}

At first, the growth factor release of the activated CM was examined in vitro with an ELISA assay. The activation led to a statistical significant increase of VEGF vs. their native control group for all tested membranes (ANOVA testing $p<$ 0.05 ) and showed growth factor levels comparable to PRF alone (for details please see Supplementary data and Fig. 1a, b). The release was significantly the highest after $24 \mathrm{~h}$ for all respective membranes in comparison to the other tested time points (post hoc test, $p<0.05$ ), but no significant difference among the tested CM could be found (ANOVA testing $p>0.05)$. Similar results were found for the release

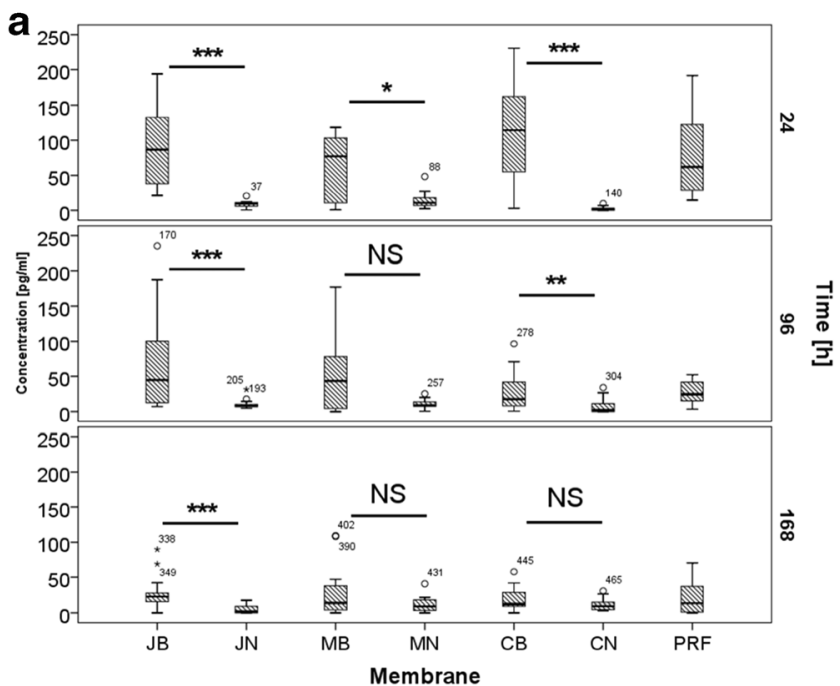

Fig. 1 Growth factor release analyzed by ELISA for (a) the VEGF and (b) the TGF expression for three different time points $(24,96,168 \mathrm{~h})$. Highest levels were reached after $24 \mathrm{~h}$, all activated membranes of TGF- $\beta$ of the respective membrane vs. native control group (ANOVA testing $p<0.05$ ) with the highest after $24 \mathrm{~h}$ (post hoc test, $p<0.001$ ). Analogous, there was no statistical significant difference among the activated groups and in comparison to the PRF group (ANOVA testing $p>0.05$ ).

\section{YSM assay}

Due to the results of the in vitro analysis, that showed maximum growth factor release after $24 \mathrm{~h}$, the membranes were incubated $24 \mathrm{~h}$ in the YSM assay in order to measure angiogenic potential at the highest value of growth factor expression. Microscopic analysis (Fig. 2i) of vessels per $\mathrm{mm}^{2}$ showed that the YSM treated with biologized membranes induced statistically significant more vessels in comparison to the native membranes and in comparison to the YSM group as negative control (each $p<0.001$ ). Between the native membranes, there was no significant difference of vessel formation after $24 \mathrm{~h}(p>0.05)$. In addition, no statistical significant difference could be found between the native membranes, PRF alone and the activated membranes (each $p>0.05$ ) and between the different activated membranes (each $p>0.05$ ).

Microscopic analysis of branching points per $\mathrm{mm}^{2}$ showed comparable results (Fig. 2j) with a significant increase of branching points per $\mathrm{mm}^{2}$ in the YSM treated with the biologized groups and the PRF (each $p<0.001$ ). Again, no statistical significant difference between the native membranes, PRF vs. the activated membranes and among the biologized membranes could be found (each $p>0.05$ ). In addition, there was a statistical significant increase of branching points per $\mathrm{mm}^{2}$ in the YSM group in comparison to the native membranes $(p<0.01)$.

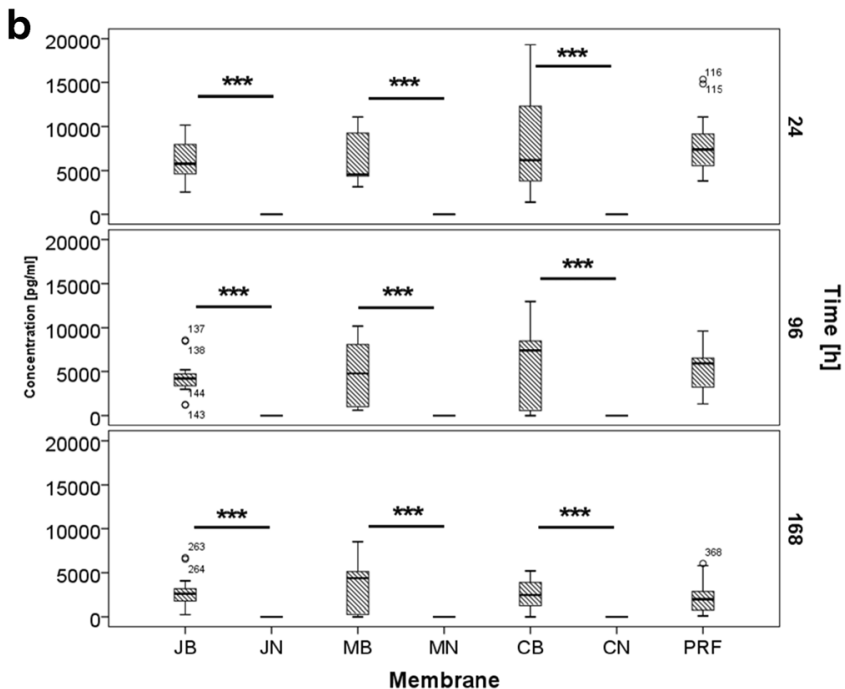

demonstrated a significant higher expression than their native pendant. For group description see manuscript. $* p<0.05$, **p $p<0.01$, *** $p<$ $0.001, \mathrm{NS}=$ no statistical significant difference 


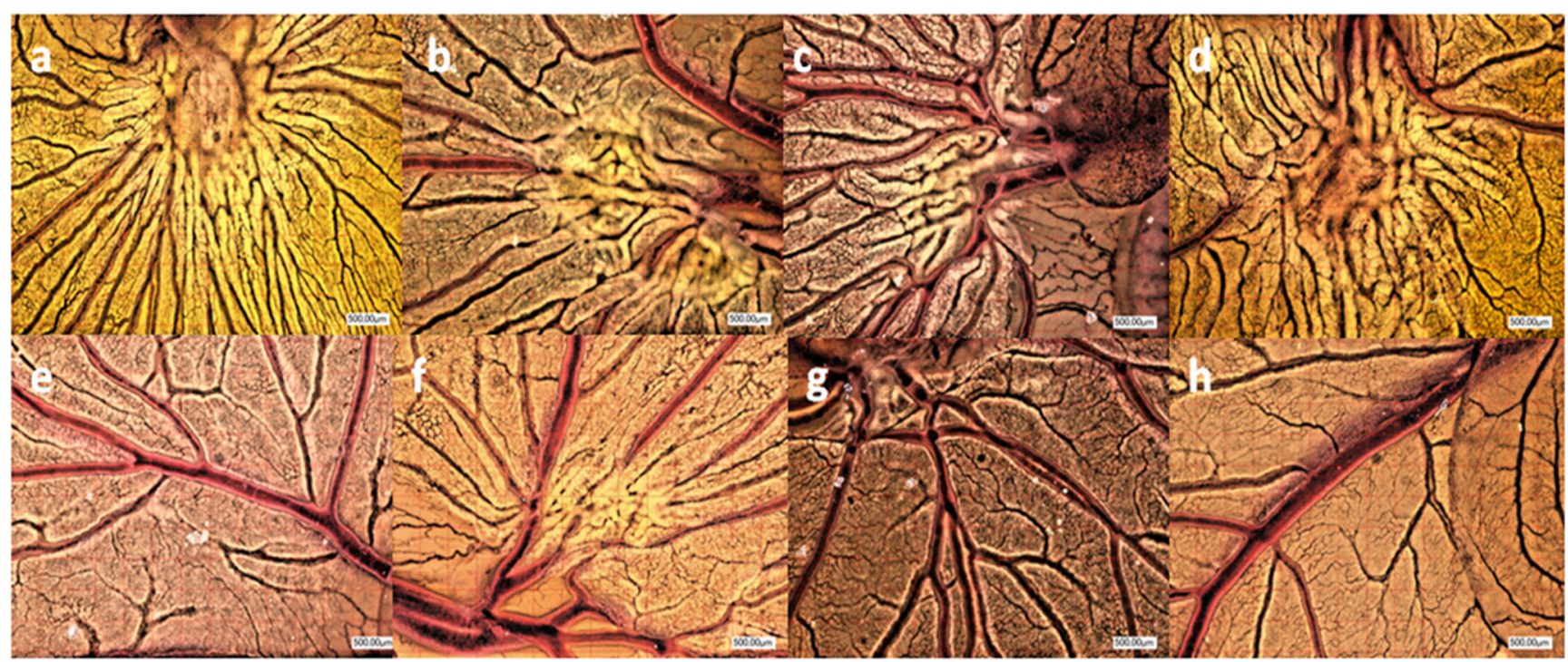

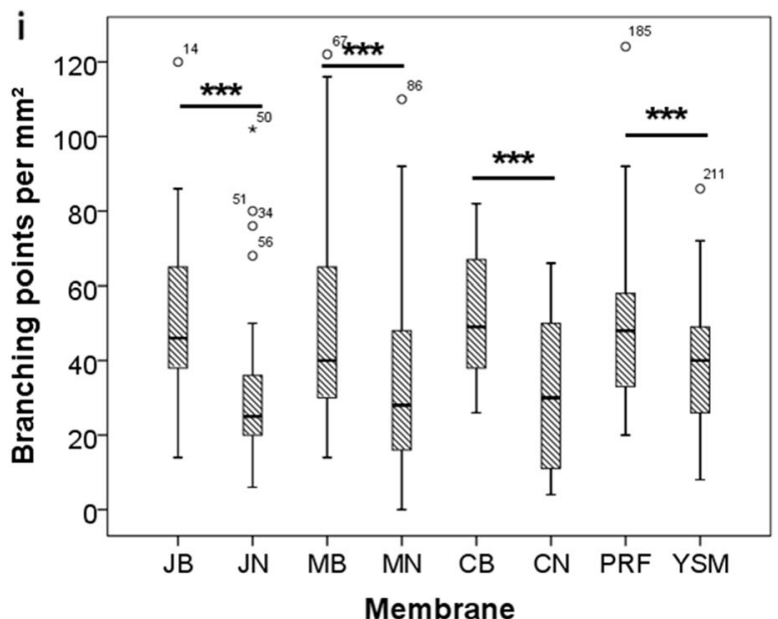

Fig. 2 Microscopic analysis of the YSM assay with (a) JB, (b) MB, (c) $\mathrm{CB}$, (d) PRF, (e) JN, (f) MN, (g) CN and (h) of a native YSM. Note the morphologic difference of the vessel network of the YSM treated with the biologized membranes (a-d) in comparison with their native pendants (e-

\section{Immunohistochemical analysis}

To verify the results, a subgroup of the YSM assay was further examined immunohistochemically with HE, $\alpha$-SMA, and CD105 staining.

The number of vessels per $\mathrm{mm}^{2}$ in the YSM treated with bioactivated membranes and PRF respectively was increased significantly (Fig. 3g, $p<0.05$ ) in comparison with their control groups. A statistical significant difference between PRF and activated membrane was found for MB $(p<0.05)$ but none for the other membranes. There was no difference between the native membranes.

For $\alpha$-SMA, the number of vessels per $\mathrm{mm}^{2}$ was significant increased in the YSM treated with the bioactivated groups (Fig. $3 \mathrm{~h}, p<0.05$ ) compared to their control groups.

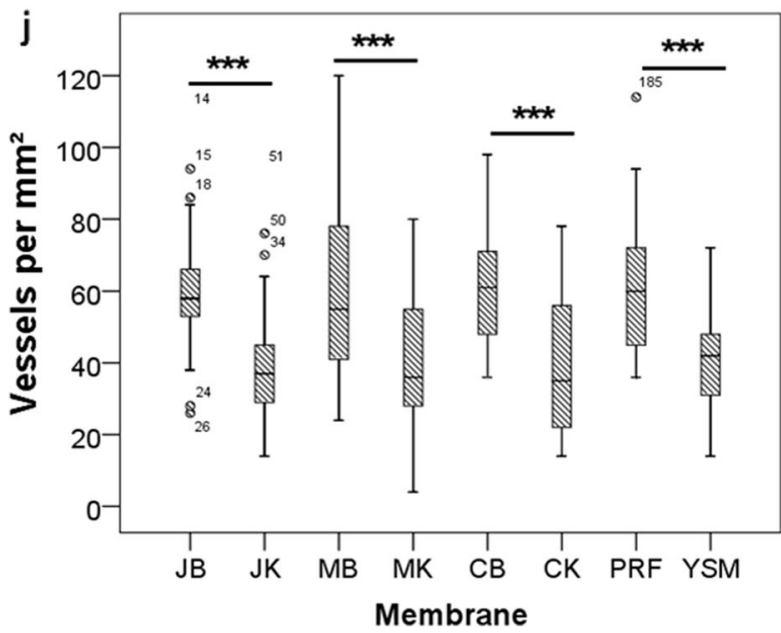

h). $n=28$ per group were evaluated. (i) Vessels per $\mathrm{mm}^{2}$. (j) Branching points per $\mathrm{mm}^{2}$. For group description see manuscript. Bars, $500 \mu \mathrm{m}$. $* p<0.001$

In contradiction to the HE staining results, there was an increase in $\alpha$-SMA+ vessels in the CB group in comparison to the JB (each $p<0.05$ ) and in comparison to the PRF (each $p<$ $0.01)$.

The immunohistochemical evaluation of the CD105+ vessels per $\mathrm{mm}^{2}$ showed an increase of vessels in all bioactivated groups, and PRF respectively, in comparison to their control groups (Fig. 3I, $p<0.05$ ). Furthermore there were significant differences between the groups: $\mathrm{CD} 105+$ vessels were increased in the MB group in comparison with the JB $(p<0.01)$, vs. the $\mathrm{CB}$ group $(p<$ $0.001)$ and the PRF group $(p<0.001)$ and an increase in the JB group in comparison with the PRF $(p<0.05)$, as well as an increase in the $\mathrm{CB}$ group compared with the PRF $(p<0.01)$. 

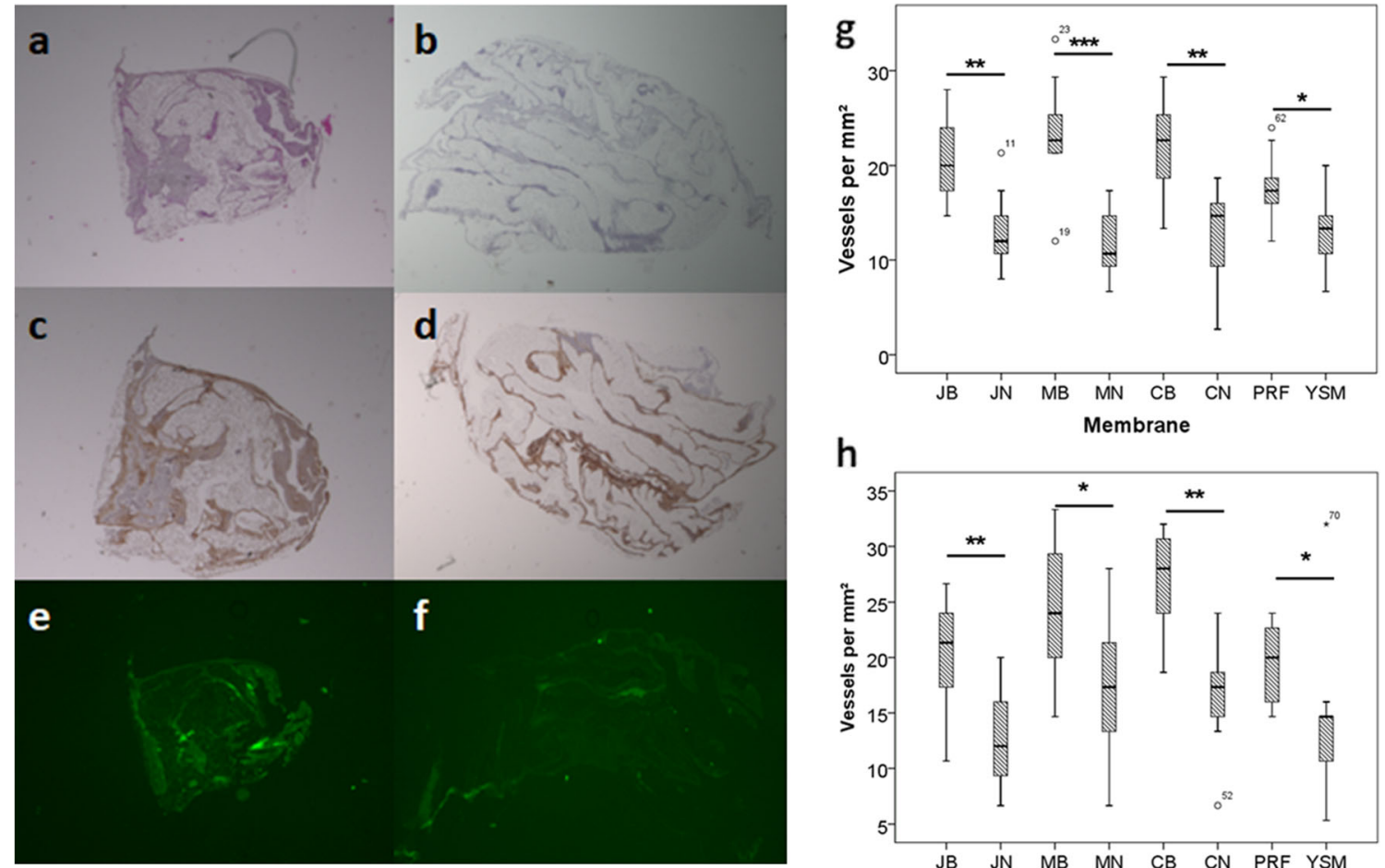

h

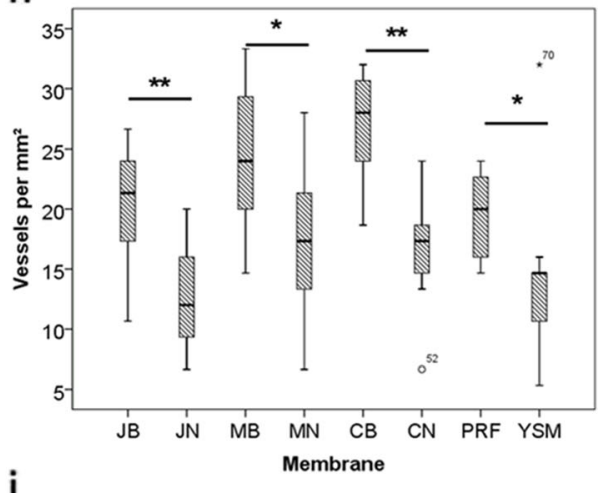

i

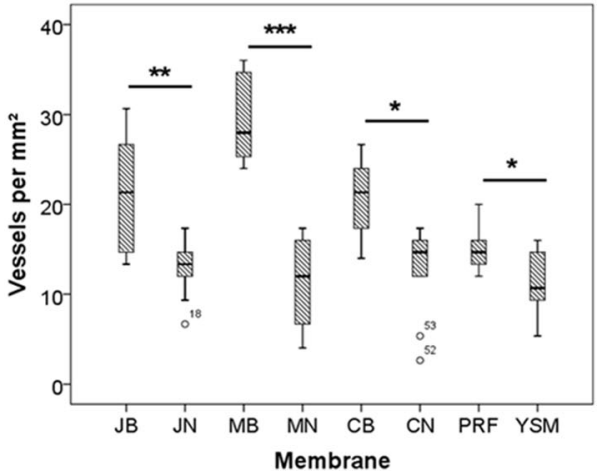

Fig. 3 Microscopic analysis of the YSM showing the HE (a,b), the $\alpha$-SMA (c,d) and the CD105 (e,f) staining. (g) Vessels per mm ${ }^{2}$ with HE, with $\alpha$ SMA (h) and CD105 (i) respectively, $n=9$ per group. For group description see manuscript. * $p<0.05$, ** $p<0.01, * * * p<0.001$

\section{Intravital fluorescence microscopy}

Additionally, pro-angiogenic potential was examined using intravital fluorescence microscopy. After FITC dextran injection, the vessels per $\mathrm{mm}^{2}$ were increased when treated with the JB membrane $(p<0.001)$ and the MB membrane respectively $(p<0.005)$ in comparison to their native controls (Fig. 4h). There was no statistical significant difference for the CAM treated with the native $\mathrm{CB}$ membrane $(p<0.2)$ and between the activated groups and the PRF group $(p>0.05)$.

There was a statistical significant increase of branching points in the JB group $(p<0.001)$ and in the MB group $(p<0.01)$ (Fig. 4i). Again, there was no statistical significant difference between the $\mathrm{CAM}$ treated with $\mathrm{CB}$ in comparison to $\mathrm{CN}(p=0.1)$. As well there was no difference between the activated membrane groups in comparison to the PRF group $(p>0.05)$.

There was an increase of vascular density in the CAM treated with the $\mathrm{MB}(p<0.05)$ and $\mathrm{CB}(p<0.05)$ in comparison to their native controls (Fig. 4j). There was no statistical significant difference between the JB and the JN group $(p<$ 0.2 ) and between the bioactivated membrane groups and the PRF $(p>0.05)$.

\section{Discussion}

In this study, it was demonstrated that PRF can be used to activate $\mathrm{CM}$ and enhance angiogenesis and vascularization in vivo and in vitro. Growth factor releases of the activated membranes were close approximately at the same level of 

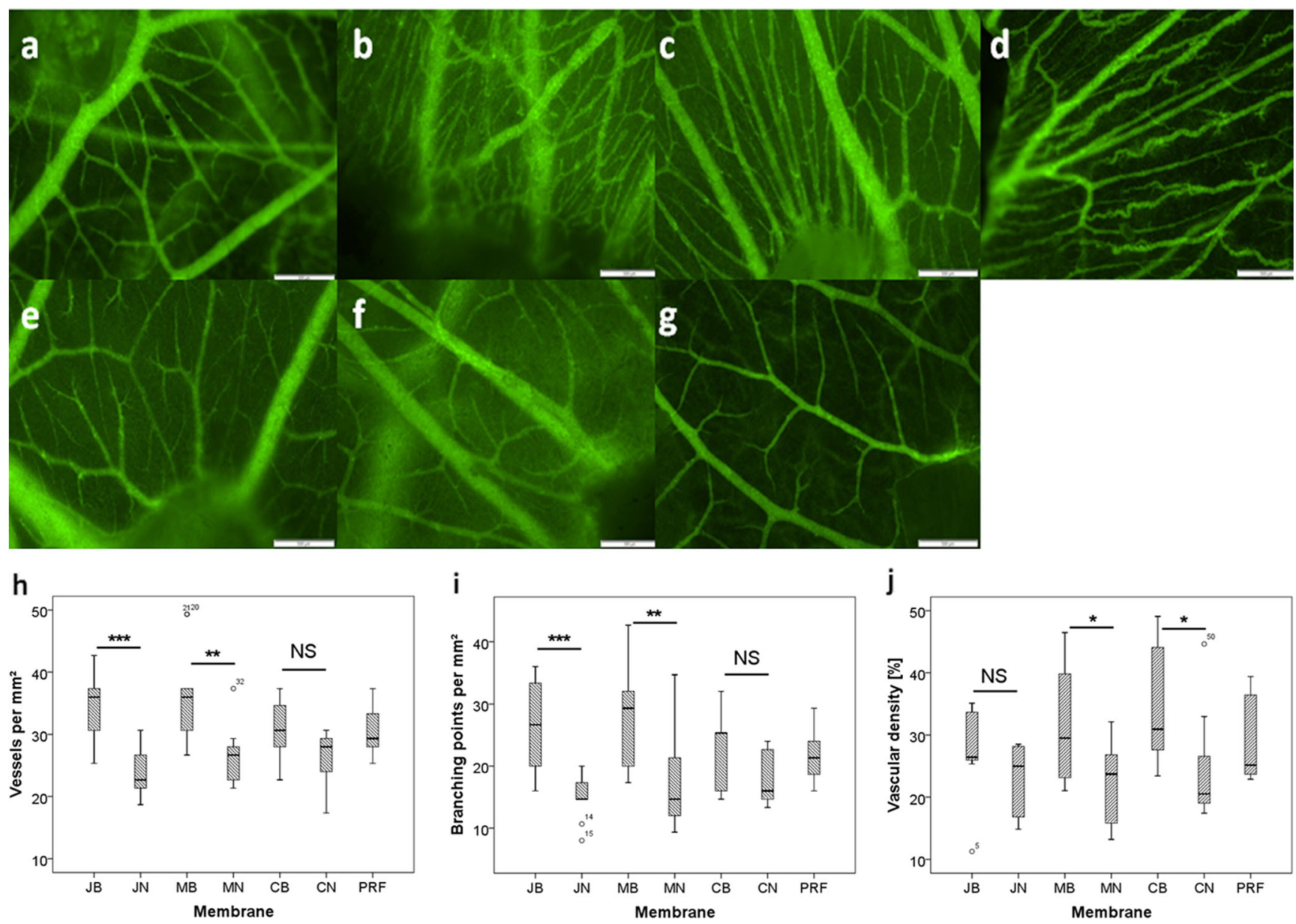

Fig. 4 In vivo fluorescent microscopic analysis of the CAM after injecting FICS-dextran with (a) JB, (b) MB, (c) CB, (d) PRF, (e) JN, (f) $\mathrm{MN}$ and $(\mathrm{g}) \mathrm{CN}$. Note the increased number of vessels in the CAMs treated with the activated membranes (a-d) as well as the denser vessel

network formed in those CAMs. (h) Vessels per $\mathrm{mm}^{2}$. (i) Braching points per $\mathrm{mm}^{2}$. (j) Vascular density. Bars, $500 \mu \mathrm{m} .{ }^{*} p<0.05$, ** $p<0.01$, *** $p<0.001, \mathrm{NS}=$ no statistical significant difference

pure PRF. In vivo, activation of CM with PRF led to increased vessel numbers, -branching points and -density.

Overall, vasculature is indispensable for appropriate tissue regeneration, repair and remodeling [22]. There are two mechanisms of blood vessel formation: de novo formation from a progenitor cells (neo-vasculogenesis) and the development from preexisting blood vessels (angiogenesis). Angiogenesis in turn comprises two different ways: endothelial sprouting, based on endothelial cell migration, and intussusceptive microvascular growth where existing vessel lumens divides by formation and insertion of tissue folds and columns of interstitial tissue into the vessel lumen [23]. Within this study, new blood vessel formation in vivo was analyzed as the primary endpoint and no differentiation between the underlying mechanisms could be performed with the used model. However, since new blood vessels seemed to develop from pre-existing ones, angiogenesis was hypothesized to be the main mechanism.
These processes are mediated by different of growth factors and chemokines where VEGF plays a distinctive role [24]. Today it is known that platelets, besides their importance at early stages in wound healing (clot formation, direct release of growth factors), can influence the whole process of tissue regeneration by modulating synthesis and release of VEGF [25]. In detail, platelets contain large amounts of pro- and anti-angiogenic proteins in $\alpha$-granule populations. When activated, their release as well as platelet-derived phospholipids and microparticles show synergistic regulatory effects on angiogenesis [26]. However, recent studies found platelet concentrates superior to plasma or platelets alone in terms of stimulating fibroblast proliferation and migration, chemotaxis and angiogenesis as relevant processes for wound healing [27]. This provides insights into the clinical relevance of platelet-derived products such as PRF for regenerative procedures [25]. In this study, the CAM and YSM assay was used to evaluate and quantify angiogenesis. Both consist of an extra- 
embryonic membrane, comprised of a high density of blood and lymphatic vessels. In this context, the YSM was described as a suitable model for characterization of angiogenic agents with comparable properties to the CAM assay [28]. Therefore, their dense capillary network is commonly used to study in vivo angiogenesis [29]. In recent a study by Ratajczak et al., the CAM assay was used to evaluate the effect of PRF on angiogenesis. The authors could demonstrate an increased number of blood vessels when treated with PRF which is in accordance with the results obtained in this study. Furthermore, a human-derived fibrin gel was able to induce blood vessels in vivo as well. This strengthens the hypothesis that the fibrin framework within the PRF may strongly contribute to angiogenesis [30].

A plentitude of studies suggest that PRF may be used for optimized soft tissue healing in regenerative medicine and periodontology $[10,11]$ and, in addition, may even show antimicrobial capacity namely against periopathogens such as Porphyrymona gingivalis [31]. However, a recent cochrane review defines the evidence for the use of PRF for treating intrabony defects insufficient [32]. Therefore, in vitro studies that define biological characteristics of the PRF are much in need that may lead the path for future clinical implantation.

Consequently, biological characteristics and release mechanism of growth factors of the PRF are of great interest. In this context, accumulated growth factor release of PRF was found up to 10 days after first incubation with the significantly highest levels at 1,3 , and 5 days for TGF $1 \beta$ and VEGF concentrations. The results further indicate the highest levels of TGF $1 \beta$ and for VEGF between $8 \mathrm{~h}$ and $24 \mathrm{~h}$ when analyzed for each time point [33]. In concordance, a maximum of growth factor release was likewise seen after $24 \mathrm{~h}$ in the presented study, but dropped relatively fast after $96 \mathrm{~h}$ and $168 \mathrm{~h}$. This demonstrates the stated gradual release mechanism of growth factors in PRF [19]. Mimicking, compacting and overlapping the physiological phases of wound healing, PRF may seen a possible tool to optimize early initiation of angiogenesis. The presented results could demonstrate an enhanced angiogenesis and vascularization of activated $\mathrm{CM}$ with PRF. In vitro, expression patterns did not change significantly when PRF was used to activate CM in comparison to the PRF clot alone.

On the one hand, growth factor release and cell content may differ inter-individually for PRF production. On the other hand, production of PRF may rely heavily on technical aspects. Today, a variety of table-top centrifuges are commercially available for the production of different PRF protocols. However, a recent study by Dohan Ehrenfest et al. found different profiles of vibrations for each centrifuge that, depending on the rotational speed, and the centrifuge characteristics are directly impacting the architecture and cell content of PRF. As a conclusion, the authors displayed the system used in this study inferior in terms of cell content, growth factor release and fibrin architecture [34]. In contradiction, Miron et al. recently demonstrated that the used centrifugation tubes had a much greater impact on the final size outcome of PRF clots in comparison to the centrifugation devices. Interestingly, the system used in this study showed, in general, significantly greater-sized clots when compared to other commercially available tubes [35]. In this context, new evidence questions the use of silica-coated plastic tubes. In a study by Tsujino et al., levels of silica microparticles were found to be incorporated into the PRF-like matrix. Therefore such PRFlike matrices negatively influence tissue regeneration through induction of inflammation and may even promote cytotoxic effects. As a conclusion the authors strongly recommend to only use the conventional types of plain glass tubes for PRF as used in this study [36]. In summary, different PRF protocols are extensively discussed in the literature with special remark to the centrifugation process. However, which tubes should be used for centrifugation process is still a controversial debate due to the weak evidence so far. After platelets and leukocytes within the fibrin scaffold were histologically proven to accumulate mainly in the proximal portion of the PRF clot, the reduction of the applied relative centrifugation force influenced the cellular distribution within the solid PRF matrix and resulted in further developed PRF concept that showed enhanced platelets and leukocytes, and there is emerging evidence that cell distribution may involve the entire clot compared to earlier PRF protocols [37]. However, their distribution seem to follow a three-dimensional pattern that may strongly relay on the centrifugation process [38].

In the present study, a stable PRF membrane was used to biologically functionalize CM. This was out of two main reasons. First, in mechanical and degradation analyses, the stable PRF membrane showed sufficient stability for the indication as a bioactive barrier membrane for alveolar bone tissue regeneration $[39,40]$. Secondly, it was shown that it resulted in a better distribution of platelets throughout the PRF matrix in the upper $4-5 \mathrm{~mL}$ and is therefore clinically recommended for membrane fabrication. In contradiction, liquid PRF was proven to be ineffective at separating cell types or producing high yields of platelets/ leukocytes [16].

Up to date, no elusive conclusion can be drawn which PRF protocol or centrifugation process is considered to have the highest quality and quantity of cell content, growth factor release or fibrin architecture. Future studies should, beside the commercially heavily triggered discussion, focus on accurate methods to validate the different protocols.

Guided tissue and guided bone regeneration (GTR/ GBR) is widely used in regenerative procedures [41]. Beside autologous transplants like connective tissue grafts or free mucosal grafts, commercial available xenograft collagen matrices represent a valide alternative to enhance soft tissue. Advantages are seen in the prevention of donor 
site morbidity and limitless availability [42]. However, there are some restrictions in the clinical workflow. A recent review found soft tissue complications after GBR such as membrane exposure, soft tissue dehiscence, and acute infection/abscess rate up to $16 \%$ [43]. As a major limitation for sufficient soft tissue healing, the restricted vascularization is discussed to play a major role [44-46]. A biological active membrane, e.g. by the transfer of the biological characteristics of autologous platelet concentrates to the membrane could overcome this limitation [41].

Today, CM represents a clinical tool for GTR procedures where a positive effect on probing depth reduction, clinical attachment gain, and percent of bone fill could be achieved. As a barrier, CM prevents epithelial down-growth along the root surfaces during the early phase of wound healing [47]. This effect may explain the results of this study obtained in the YSM model where native collagen matrices showed lower numbers of vessels and branching points in comparison to the untreated YSM. All three CM analyzed in this study are frequently used in clinical routine for soft and hard tissue regeneration and thickening procedures around teeth and dental implants with a significant benefit on keratinized mucosa width augmentation 4 weeks after implant insertion demonstrated [48]. Furthermore, a long-lasting barrier function with slow biodegradation for the porcine pericardium membrane could be shown in vitro [4]. Furthermore, they exhibit different mechanical behaviors. Ortolani et al. demonstrated differences between pericard (jason $®$ ) and dermis (collprotect $\mathbb{B}$ ) dervied membranes that proved the pericard derived membran the stiffest one with an impaired elastic module but with highest strength. Instead, Collprotect specimens displayed minimal differences in terms of elastic modulus, maximum tensile stress and stress relaxation in the middle ranges [49]. From a mechanical site of view, the thin pericard derived membrane may retain platelets [50] and other component of the PRF in the activation process, but it could be hypothesized that the thicker matrix of the other two tested membrane may be further loaded and therefore facilate biofunctionalization. However, the in vitro results obtained in this study demonstrated no significant differences in growth factor release kinetics of the native membranes nor in in vivo angiogenic properties except for CD105+ staining and for vessel numbers and density after FITC cextran injection for mucoderm. This underlines the assumption that a thicker matrix facilitates biofunctionalization-process via PRF.

For the non cross-linked collagen scaffolds, animal studies revealed only a slightly differentiated biodegradation pattern in terms of presence of inflammatory cells and cell invasion in comparison to cross linked collagen membranes [51]. In addition, it could be shown that in vivo acellular dermal matrices are capable of significant revascularization of its collagen structure in the early healing period [52].
Kasaj et al. evaluated the impact of rehydration protocols on biomechanical and structural properties of different acellular collagen matrices and concluded that rehydration protocol affects the biomechanical properties of ACMs, such as mucoderm ${ }^{\circledR}$ membrane significantly [53]. It will be interesting to analyze in future studies, if stable or liquid PRF, despite their biological properties, may be useful for rehydration of the $\mathrm{CM}$ to preserve tissue matrix integrity.

A recent review that evaluated adsorption and release kinetics of growth factors on barrier membranes for guided tissue/bone regeneration showed similar results to the presented study with an early burst of growth factors [54]. Ex vivo analysis revealed that PRF is able to penetrate CM in approximately $15 \mathrm{~min}$ [55]. A recent study demonstrated the effect of a 24 h-PRF exudate modification of a porcine-derived collagen matrix. It promoted significantly activity and migration of human endothelial cells in vitro compared with serum-free medium controls [8].

In this study, overall, no difference between the respective matrices could be found. However, the in vivo experiments displayed better results in terms of vessel numbers and density in favor for mucoderm and collprotect matrices. A possible explanation may be seen in the fact that via the $3 \mathrm{D}$ architecture host cell migration and penetration could be enhanced by its interconnected structure that may lead to an increased microvessel formation and therefore angiogenesis $[20,48]$. However, future in vivo studies are needed to reveal a possible superiority from one bio-functionalized CM that can be recommended for clinical use.

In the literature, there are a plentitude of studies comparing PRF with different membranes for clinical application [56]. Contrary, there is little literature dealing with relevant information about the combination and biofunctionalization of $\mathrm{CM}$ and PRF-membranes. Some case reports stated PRF activated collagen matrices as a promising solution for sinus floor augmentation [57] or closure of oro-antral fistulas [58]. Ansarizadeh et al. recently described the mechanistic properties of collagen-chitosan membrane activated with lyophilized PRF. They found a decreased degradation rate of the membranes from 90 to $20 \%$ after 4 weeks and furthermore an effect of the membrane on osseous differentiation in vitro [59]. Consequently, the approach described in this manuscript should be further evaluated on mechanical characters such as degradation rate and a possible effect on osteoblast differentiation and proliferation (and therefore hard tissue regeneration) in future studies.

In summary, the presented study introduces and evaluates a possible methodology to use PRF to active CM biologically that can accelerate angiogenesis especially in the early woundhealing phase. It therefore represents a promising approach to enhance soft tissue regeneration. This leads the way to further clinical trials that evaluate this approach and the possible implementation in the clinical workflow. 
Funding information Open Access funding provided by Projekt DEAL. This work was supported by an intramural funding of the Platform for Biomaterial Research, Biomatics Group, University Medical Center, Langenbeckstrasse 1, 55131, Mainz, Germany.

\section{Compliance with ethical standards}

Conflict of interest Eik Schiegnitz, Bilal Al-Nawas received speaker fees from Straumann, Dentsply and Geistlich, Keyvan Sagheb received speaker fees from Straumann, Peer Kämmerer received speaker fees and research support from Straumann, Andreas Pabst received speaker fees and research support from Straumann and Botiss.

The other authors declare that they have no conflict of interest.

Ethical approval All procedures performed in studies involving human participants were in accordance with the ethical standards of the national research committee (Ärztekammer Rheinland-Pfalz) and with the 1964 Helsinki declaration and its later amendments or comparable ethical standards.

Informed consent Informed consent was obtained from all individual participants included in the study.

Open Access This article is licensed under a Creative Commons Attribution 4.0 International License, which permits use, sharing, adaptation, distribution and reproduction in any medium or format, as long as you give appropriate credit to the original author(s) and the source, provide a link to the Creative Commons licence, and indicate if changes were made. The images or other third party material in this article are included in the article's Creative Commons licence, unless indicated otherwise in a credit line to the material. If material is not included in the article's Creative Commons licence and your intended use is not permitted by statutory regulation or exceeds the permitted use, you will need to obtain permission directly from the copyright holder. To view a copy of this licence, visit http://creativecommons.org/licenses/by/4.0/.

\section{References}

1. Bottino MC, Thomas V (2015) Membranes for periodontal regeneration-a materials perspective. Frontiers of oral biology 17: 90-100. https://doi.org/10.1159/000381699

2. Pabst AM, Lehmann KM, Walter C, Kruger M, Stratul SI, Kasaj A (2016) Influence of porcine-derived collagen matrix on endothelial progenitor cells: an in vitro study. Odontology 104(1):19-26. https://doi.org/10.1007/s10266-014-0186-x

3. Maurer T, Stoffel MH, Belyaev Y, Stiefel NG, Vidondo B, Küker S, Mogel H, Schäfer B, Balmer J (2018) Structural characterization of four different naturally occurring porcine collagen membranes suitable for medical applications. PLoS One 13(10):e0205027. https:// doi.org/10.1371/journal.pone.0205027

4. Rothamel D, Schwarz F, Fienitz T, Smeets R, Dreiseidler T, Ritter L, Happe A, Zoller J (2012) Biocompatibility and biodegradation of a native porcine pericardium membrane: results of in vitro and in vivo examinations. Int J Oral Maxillofac Implants 27(1):146154

5. Saghiri MA, Asatourian A, Garcia-Godoy F, Sheibani N (2016) The role of angiogenesis in implant dentistry part II: the effect of bone-grafting and barrier membrane materials on angiogenesis. Medicina oral, patologia oral y cirugia bucal 21(4):e526-e537. https://doi.org/10.4317/medoral.21200
6. Kammerer PW, Palarie V, Schiegnitz E, Nacu V, Draenert FG, AlNawas B (2013) Influence of a collagen membrane and recombinant platelet-derived growth factor on vertical bone augmentation in implant-fixed deproteinized bovine bone-animal pilot study. Clin Oral Implants Res 24(11):1222-1230. https://doi.org/10. $1111 / j .1600-0501.2012 .02534 . x$

7. Del Corso M, Vervelle A, Simonpieri A, Jimbo R, Inchingolo F, Sammartino G, Dohan Ehrenfest DM (2012) Current knowledge and perspectives for the use of platelet-rich plasma (PRP) and platelet-rich fibrin (PRF) in oral and maxillofacial surgery part 1: periodontal and dentoalveolar surgery. Curr Pharm Biotechnol 13(7):1207-1230

8. Park JS, Pabst AM, Ackermann M, Moergel M, Jung J, Kasaj A (2018) Biofunctionalization of porcine-derived collagen matrix using enamel matrix derivative and platelet-rich fibrin: influence on mature endothelial cell characteristics in vitro. Clin Oral Investig 22(2):909-917. https://doi.org/10.1007/s00784-0172170-7

9. Choukroun J, Diss A, Simonpieri A, Girard MO, Schoeffler C, Dohan SL, Dohan AJ, Mouhyi J, Dohan DM (2006) Platelet-rich fibrin (PRF): a second-generation platelet concentrate. Part IV: clinical effects on tissue healing. Oral surgery, oral medicine, oral pathology, oral radiology, and endodontics 101(3):e56-e60. https:// doi.org/10.1016/j.tripleo.2005.07.011

10. Padma R, Shilpa A, Kumar PA, Nagasri M, Kumar C, Sreedhar A (2013) A split mouth randomized controlled study to evaluate the adjunctive effect of platelet-rich fibrin to coronally advanced flap in Miller's class-I and II recession defects. Journal of Indian Society of Periodontology 17(5):631-636. https://doi.org/10.4103/0972124x.119281

11. Mehta DB, Deshpande NC, Dandekar SA (2018) Comparative evaluation of platelet-rich fibrin membrane and collagen membrane along with demineralized freeze-dried bone allograft in grade II furcation defects: a randomized controlled study. Journal of Indian Society of Periodontology 22(4):322-327. https://doi.org/10.4103/ jisp.jisp_310_17

12. Daugela P, Grimuta V, Sakavicius D, Jonaitis J, Juodzbalys G (2018) Influence of leukocyte- and platelet-rich fibrin (L-PRF) on the outcomes of impacted mandibular third molar removal surgery: a split-mouth randomized clinical trial. Quintessence international (Berlin, Germany : 1985) 49(5):377-388. https://doi.org/10.3290/j. qi.a40113

13. Oncu E, Alaaddinoglu EE (2015) The effect of platelet-rich fibrin on implant stability. The international journal of oral \& maxillofacial implants 30(3):578-582. https://doi.org/10.11607/jomi.3897

14. Talaat WM, Ghoneim MM, Salah O, Adly OA (2018) Autologous bone marrow concentrates and concentrated growth factors accelerate bone regeneration after Enucleation of mandibular pathologic lesions. The Journal of craniofacial surgery 29(4):992-997. https:// doi.org/10.1097/scs.0000000000004371

15. Park JH, Kim JW, Kim SJ (2017) Does the addition of bone morphogenetic protein 2 to platelet-rich fibrin improve healing after treatment for medication-related osteonecrosis of the jaw? Journal of oral and maxillofacial surgery 75(6):1176-1184. https://doi.org/ 10.1016/j.joms.2016.12.005

16. Miron RJ, Chai J, Zheng S, Feng M, Sculean A, Zhang Y (2019) A novel method for evaluating and quantifying cell types in platelet rich fibrin and an introduction to horizontal centrifugation. $\mathrm{J}$ Biomed Mater Res A 107(10):2257-2271. https://doi.org/10. 1002/jbm.a.36734

17. Beck L Jr, D'Amore PA (1997) Vascular development: cellular and molecular regulation. FASEB journal 11(5):365-373

18. Herrera-Vizcaino C, Dohle E, Al-Maawi S, Booms P, Sader R, Kirkpatrick CJ, Choukroun J, Ghanaati S (2019) Platelet-rich fibrin secretome induces three dimensional angiogenic activation in vitro. 
European cells \& materials 37:250-264. https://doi.org/10.22203/ eCM.v037a15

19. Miron RJ, Zucchelli G, Pikos MA, Salama M, Lee S, Guillemette V, Fujioka-Kobayashi M, Bishara M, Zhang Y, Wang HL, Chandad F, Nacopoulos C, Simonpieri A, Aalam AA, Felice P, Sammartino G, Ghanaati S, Hernandez MA, Choukroun J (2017) Use of plateletrich fibrin in regenerative dentistry: a systematic review. Clin Oral Investig 21(6):1913-1927. https://doi.org/10.1007/s00784-0172133-z

20. Barbeck M, Lorenz J, Kubesch A, Bohm N, Booms P, Choukroun J, Sader R, Kirkpatrick CJ, Ghanaati S (2015) Porcine dermisderived collagen membranes induce implantation bed vascularization via multinucleated Giant cells: a physiological reaction? The Journal of oral implantology 41(6):e238-e251. https://doi.org/10. 1563/aaid-joi-D-14-00274

21. Choukroun J, Ghanaati S (2018) Reduction of relative centrifugation force within injectable platelet-rich-fibrin (PRF) concentrates advances patients' own inflammatory cells, platelets and growth factors: the first introduction to the low speed centrifugation concept. European journal of trauma and emergency surgery 44(1):8795. https://doi.org/10.1007/s00068-017-0767-9

22. Filipowska J, Tomaszewski KA, Niedzwiedzki L, Walocha JA, Niedzwiedzki T (2017) The role of vasculature in bone development, regeneration and proper systemic functioning. Angiogenesis 20(3):291-302. https://doi.org/10.1007/s10456-017-9541-1

23. Patan S (2000) Vasculogenesis and angiogenesis as mechanisms of vascular network formation, growth and remodeling. J NeuroOncol 50(1-2):1-15

24. Adini A, Adini I, Chi ZL, Derda R, Birsner AE, Matthews BD, D'Amato RJ (2017) A novel strategy to enhance angiogenesis in vivo using the small VEGF-binding peptide PR1P. Angiogenesis 20(3):399-408. https://doi.org/10.1007/s10456-0179556-7

25. Nami N, Feci L, Napoliello L, Giordano A, Lorenzini S, Galeazzi M, Rubegni P, Fimiani M (2016) Crosstalk between platelets and PBMC: new evidence in wound healing. Platelets 27(2):143-148. https://doi.org/10.3109/09537104.2015.1048216

26. Walsh TG, Metharom P, Berndt MC (2015) The functional role of platelets in the regulation of angiogenesis. Platelets 26(3):199-211. https://doi.org/10.3109/09537104.2014.909022

27. van der Bijl I, Vlig M, Middelkoop E, de Korte D (2019) Allogeneic platelet-rich plasma (PRP) is superior to platelets or plasma alone in stimulating fibroblast proliferation and migration, angiogenesis, and chemotaxis as relevant processes for wound healing. Transfusion. https://doi.org/10.1111/trf.15535

28. As MN, Deshpande R, Kale VP, Bhonde RR, Datar SP (2018) Establishment of an in ovo chick embryo yolk sac membrane (YSM) assay for pilot screening of potential angiogenic and antiangiogenic agents. Cell Biol Int 42(11):1474-1483. https://doi.org/ 10.1002/cbin.11051

29. Naik M, Brahma P, Dixit M (2018) A cost-effective and efficient Chick ex-Ovo CAM assay protocol to assess angiogenesis. Methods and protocols 1(2). https://doi.org/10.3390/mps1020019

30. Ratajczak J, Vangansewinkel T, Gervois P, Merckx G, Hilkens P, Quirynen M, Lambrichts I, Bronckaers A (2018) Angiogenic properties of 'Leukocyte- and platelet-rich Fibrin'. Sci Rep 8(1):14632. https://doi.org/10.1038/s41598-018-32936-8

31. Castro AB, Herrero ER, Slomka V, Pinto N, Teughels W, Quirynen M (2019) Antimicrobial capacity of leucocyte-and platelet rich fibrin against periodontal pathogens. Sci Rep 9(1):8188. https://doi. org/10.1038/s41598-019-44755-6

32. Del Fabbro M, Karanxha L, Panda S, Bucchi C, Nadathur Doraiswamy J, Sankari M, Ramamoorthi S, Varghese S, Taschieri S (2018) Autologous platelet concentrates for treating periodontal infrabony defects. The. Cochrane database of systematic reviews 11:Cd011423. https://doi.org/10.1002/14651858.CD011423.pub2
33. Kobayashi E, Fluckiger L, Fujioka-Kobayashi M, Sawada K, Sculean A, Schaller B, Miron RJ (2016) Comparative release of growth factors from PRP, PRF, and advanced-PRF. Clin Oral Investig 20(9):2353-2360. https://doi.org/10.1007/s00784-0161719-1

34. Dohan Ehrenfest DM, Pinto NR, Pereda A, Jimenez P, Corso MD, Kang BS, Nally M, Lanata N, Wang HL, Quirynen M (2018) The impact of the centrifuge characteristics and centrifugation protocols on the cells, growth factors, and fibrin architecture of a leukocyteand platelet-rich fibrin (L-PRF) clot and membrane. Platelets 29(2): 171-184. https://doi.org/10.1080/09537104.2017.1293812

35. Miron RJ, Xu H, Chai J, Wang J, Zheng S, Feng M, Zhang X, Wei Y, Chen Y, Mourao C, Sculean A, Zhang Y (2019) Comparison of platelet-rich fibrin (PRF) produced using 3 commercially available centrifuges at both high $(\sim 700 \mathrm{~g})$ and low $(\sim 200 \mathrm{~g})$ relative centrifugation forces. Clin Oral Investig:1-12. https://doi.org/10.1007/ s00784-019-02981-2

36. Tsujino T, Takahashi A, Yamaguchi S, Watanabe T, Isobe K, Kitamura Y, Tanaka T, Nakata K, Kawase T (2019) Evidence for contamination of silica microparticles in advanced platelet-rich fibrin matrices prepared using silica-coated plastic tubes. Biomedicines 7(2). https://doi.org/10.3390/biomedicines7020045

37. Ghanaati S, Booms P, Orlowska A, Kubesch A, Lorenz J, Rutkowski J, Landes C, Sader R, Kirkpatrick C, Choukroun J (2014) Advanced platelet-rich fibrin: a new concept for cell-based tissue engineering by means of inflammatory cells. The Journal of oral implantology 40(6):679-689. https://doi.org/10.1563/aaid-joiD-14-00138

38. Dohan Ehrenfest DM, Del Corso M, Diss A, Mouhyi J, Charrier JB (2010) Three-dimensional architecture and cell composition of a Choukroun's platelet-rich fibrin clot and membrane. J Periodontol 81(4):546-555. https://doi.org/10.1902/jop.2009.090531

39. Isobe K, Watanebe T, Kawabata H, Kitamura Y, Okudera T, Okudera H, Uematsu K, Okuda K, Nakata K, Tanaka T, Kawase $\mathrm{T}$ (2017) Mechanical and degradation properties of advanced platelet-rich fibrin (A-PRF), concentrated growth factors (CGF), and platelet-poor plasma-derived fibrin (PPTF). International journal of implant dentistry 3(1):17. https://doi.org/10.1186/s40729017-0081-7

40. Kardos D, Hornyak I, Simon M, Hinsenkamp A, Marschall B, Vardai R, Kallay-Menyhard A, Pinke B, Meszaros L, Kuten O, Nehrer S, Lacza Z (2018) Biological and mechanical properties of platelet-rich fibrin membranes after thermal manipulation and preparation in a single-syringe closed system. Int J Mol Sci 19(11). https://doi.org/10.3390/ijms19113433

41. Bottino MC, Thomas V, Schmidt G, Vohra YK, Chu TM, Kowolik MJ, Janowski GM (2012) Recent advances in the development of GTR/GBR membranes for periodontal regeneration-a materials perspective. Dental materials 28(7):703-721. https://doi.org/10. 1016/j.dental.2012.04.022

42. Bassetti RG, Stahli A, Bassetti MA, Sculean A (2017) Soft tissue augmentation around osseointegrated and uncovered dental implants: a systematic review. Clin Oral Investig 21(1):53-70. https://doi.org/10.1007/s00784-016-2007-9

43. Lim G, Lin GH, Monje A, Chan HL, Wang HL (2018) Wound healing complications following guided bone regeneration for ridge augmentation: a systematic review and meta-analysis. The international journal of oral \& maxillofacial implants 33(1):41-50. https:// doi.org/10.11607/jomi.5581

44. Stosich MS, Moioli EK, Wu JK, Lee CH, Rohde C, Yoursef AM, Ascherman J, Diraddo R, Marion NW, Mao JJ (2009) Bioengineering strategies to generate vascularized soft tissue grafts with sustained shape. Methods (San Diego, Calif) 47(2):116-121. https://doi.org/10.1016/j.ymeth.2008.10.013

45. Velnar T, Gradisnik L (2018) Tissue augmentation in wound healing: the role of endothelial and epithelial cells. Medical 
archives (Sarajevo, Bosnia and Herzegovina) 72(6):444-448. https://doi.org/10.5455/medarh.2018.72.444-448

46. Li J, Zhang YP, Kirsner RS (2003) Angiogenesis in wound repair: angiogenic growth factors and the extracellular matrix. Microsc Res Tech 60(1):107-114. https://doi.org/10.1002/jemt.10249

47. Bunyaratavej P, Wang HL (2001) Collagen membranes: a review. J Periodontol 72(2):215-229. https://doi.org/10.1902/jop.2001.72.2. 215

48. Papi P, Pompa G (2018) The use of a novel porcine derived Acellular dermal matrix (Mucoderm) in Peri-implant soft tissue augmentation: preliminary results of a prospective pilot cohort study. Biomed Res Int 2018:6406051. https://doi.org/10.1155/ 2018/6406051

49. Ortolani E, Quadrini F, Bellisario D, Santo L, Polimeni A, Santarsiero A (2015) Mechanical qualification of collagen membranes used in dentistry. Annali dell'Istituto superiore di sanita 51(3):229-235. https://doi.org/10.4415/ann_15_03_11

50. Gauvin R, Marinov G, Mehri Y, Klein J, Li B, Larouche D, Guzman R, Zhang Z, Germain L, Guidoin R (2013) A comparative study of bovine and porcine pericardium to highlight their potential advantages to manufacture percutaneous cardiovascular implants. J Biomater Appl 28(4):552-565. https://doi.org/10.1177/ 0885328212465482

51. Rothamel D, Benner M, Fienitz T, Happe A, Kreppel M, Nickenig HJ, Zoller JE (2014) Biodegradation pattern and tissue integration of native and cross-linked porcine collagen soft tissue augmentation matrices - an experimental study in the rat. Head Face Med 10:10. https://doi.org/10.1186/1746-160x-10-10

52. Pabst AM, Happe A, Callaway A, Ziebart T, Stratul SI, Ackermann M, Konerding MA, Willershausen B, Kasaj A (2014) In vitro and in vivo characterization of porcine acellular dermal matrix for gingival augmentation procedures. J Periodontal Res 49(3):371-381. https://doi.org/10.1111/jre.12115

53. Kasaj A, Levin L, Stratul SI, Gotz H, Schlee M, Rutters CB, Konerding MA, Ackermann M, Willershausen B, Pabst AM (2016) The influence of various rehydration protocols on biomechanical properties of different acellular tissue matrices. Clin Oral Investig 20(6):1303-1315. https://doi.org/10.1007/s00784-015$1614-1$
54. Caballe-Serrano J, Abdeslam-Mohamed Y, Munar-Frau A, Fujioka-Kobayashi M, Hernandez-Alfaro F, Miron R (2019) Adsorption and release kinetics of growth factors on barrier membranes for guided tissue/bone regeneration: a systematic review. Arch Oral Biol 100:57-68. https://doi.org/10.1016/j.archoralbio. 2019.02.006

55. Al-Maawi S, Vorakulpipat C, Orlowska A, Zrnc TA, Sader RA, Kirkpatrick CJ, Ghanaati S (2018) In vivo implantation of a bovine-derived collagen membrane leads to changes in the physiological cellular pattern of wound healing by the induction of multinucleated Giant cells: an adverse reaction? Frontiers in bioengineering and biotechnology 6:104. https://doi.org/10.3389/fbioe.2018. 00104

56. Jain A, Jaiswal GR, Kumathalli K, Kumar R, Singh A, Sarwan A (2017) Comparative evaluation of platelet rich fibrin and dehydrated amniotic membrane for the treatment of gingival recession- a clinical study. Journal of clinical and diagnostic research 11(8):Zc24-zc28. https://doi.org/10.7860/jcdr/2017/29599. 10362

57. Gulsen U, Dereci O (2019) Evaluation of new bone formation in sinus floor augmentation with injectable platelet-rich fibrin-soaked collagen plug: a pilot study. Implant Dent 28(3):220-225. https:// doi.org/10.1097/id.0000000000000883

58. Pandikanda R, Singh R, Patil V, Sharma M, Shankar K (2018) Flapless closure of oro-antral communication with PRF membrane and composite of PRF and collagen - a technical note. Journal of stomatology, oral and maxillofacial surgery. https://doi.org/10. 1016/j.jormas.2018.12.012

59. Ansarizadeh M, Mashayekhan S, Saadatmand M (2019) Fabrication, modeling and optimization of lyophilized advanced platelet rich fibrin in combination with collagen-chitosan as a guided bone regeneration membrane. Int J Biol Macromol 125:383391. https://doi.org/10.1016/j.ijbiomac.2018.12.078

Publisher's note Springer Nature remains neutral with regard to jurisdictional claims in published maps and institutional affiliations. 